\title{
Morfometria e crescimento inicial de Copaifera pubiflora exposta à terra vegetal produzida em compostagem
}

\author{
Carolina Soares Marquesa ${ }^{a}$, Pedro Vitor Pereira Guimarães ${ }^{b}$, Oscar José Smiderlec, Rita de Cássia Pompeu de \\ Sousa $^{d}$ \\ a Laboratório de Análise de Sementes, Universidade Federal de Roraima, Boa Vista, 69310-000, Roraima, Brasil, * carolinasoaresmarques@hotmail.com. \\ b Programa de Pós-Graduação em Agroecologia, Universidade Estadual de Roraima, Boa Vista, 69306-530, Roraima, Brasil. \\ c Laboratório de Análise de Sementes, Embrapa Roraima, Boa Vista, 69301-970, Roraima, Brasil. \\ ' Laboratório de Resíduos, Embrapa Roraima, Boa Vista, 69301-970, Roraima, Brasil.
}

Recebido: 07 dezembro 2017 / Aceito: 08 janeiro 2018 / Publicado online: 20 janeiro 2018

\begin{abstract}
Resumo
Essa pesquisa objetivou avaliar a emergência e o crescimento inicial de Copaifera pubiflora Benth. (copaibeira) expostas à terra vegetal produzida em compostagem escolar. As sementes de $C$. pubiflora foram coletadas em área de mata ciliar de Boa Vista, Roraima e classificadas quanto o tamanho (pequenas ou grandes) e realizada a caracterização biométrica (comprimento, diâmetro e massa da semente). O experimento consistiu de seis diferentes doses de terra vegetal $(0,20,40$, 60,80 e 100\%) em areia esterilizada. As várias respostas avaliadas foram: porcentagem de emergência de plântulas, altura da parte aérea, diâmetro do coleto e matéria seca de plântulas. Houve diferença significativa na qualidade de mudas de $C$. pubiflora cultivadas em função do tamanho de sementes e doses de terra vegetal. As plantas oriundas de sementes grandes apresentam melhor qualidade nas variáveis mensuradas. O uso de terra vegetal em doses maiores que 20 e $40 \%$ interferem negativamente na emergência de sementes pequenas e grandes de $C$. pubiflora, respectivamente. $\mathrm{O}$ crescimento inicial de plantas de $C$. pubiflora é beneficiado quando se utiliza doses de até $60 \%$ de terra vegetal e $40 \%$ de areia esterilizada, para ambos tamanhos de sementes.
\end{abstract}

Palavras-chave: Biometria de sementes, Espécie florestal nativa, Substrato orgânico.

\section{Morphometry and initial growth of Copaifera pubiflora exposed to vegetable land produced in composting}

\begin{abstract}
This research aimed to assess the emergence and initial growth of Copaifera pubiflora Benth. (copaibeira) exposed to vegetable land produced in school composting. The seeds of $C$. pubiflora were collected in the area of the riparian forest of Boa Vista, Roraima and classified as size (small or large) and performed the biometric characterization (length, diameter and mass of the seed). The experiment consisted of six different doses of vegetable land $(0,20,40,60,80$ and 100\%) in sterile sand. The variables evaluated responses were: emergency percentage of seedlings, height of the aerial part, diameter of the stem and dry matter of seedlings. There was significant difference in the quality of seedlings of $C$. pubiflora cultivated according to the size of seeds and doses of vegetable land. Plants originating from large seeds have better quality in the measured variables. The use of vegetable land in doses greater than 20 and $40 \%$ interferes negatively in the emergence of small and large seeds of $C$. pubiflora, respectively. The initial growth of plants from C. pubiflora is benefited when using doses of up to $60 \%$ of vegetable land and $40 \%$ of sterile sand, for both seed sizes.
\end{abstract}

Keywords: Seed biometry, Native forest species, Organic substrate.

\section{Introdução}

As espécies do gênero Copaifera L. (Caesalpiniaceae) são encontrados em todos os trópicos, sendo o Brasil, país com maior incidência, onde 16 espécies tem ampla distribuição (Veiga Junior e Pinto, 2002). As plantas de $C$. publifora atingem cerca de 40 metros de altura, 140 centímetros de diâmetro ou rodo de até 4 metros, possuem casca aromática, folhagem densa, flores pequenas e frutos secos, do tipo vagem. As sementes são pretas e ovoides com um arilo amarelo rico em lipídeos (Shanley, 1996; Martins-Silva et al., 2008).

A C. pubiflora é uma espécie com ampla distribuição no norte da América do Sul, ocorre no Brasil, Colômbia, 
Guiana e na Venezuela. Na Amazônia brasileira, só foi coletada no extremo norte, no estado de Roraima, onde está amplamente distribuída. Habita várzeas e savanas, podendo também ser encontrada em matas de terra firme. (Martinsda-Silva et al., 2008). Em Roraima, a C. pubiflora é a principal espécie produtora de óleo resina do gênero Copaifera (Shanley, 1996).

Dentre as inúmeras espécies com potencial na região Amazônica, a $C$. pubiflora pode ser inserida em programas de recomposição de mata ciliar, recuperação de área degradadas, bem como de consórcios em sistemas agroflorestais. No entanto, para obtenção de mudas com qualidade é necessário que emergência de plântula e desenvolvimento inicial da planta, aconteça de forma eficiente e eficaz. Uma das dificuldades enfrentadas na produção de mudas é o crescimento lento apresentado por muitas espécies florestais. Em face disso, torna-se importante a definição de estratégias que favoreçam a sua produção com qualidade e menor tempo (Ros et al., 2005).

A obtenção de um substrato eficiente para formação e produção de mudas florestais, deve apresentar certas características desejáveis, tais como: disponibilidade de aquisição na região, facilidade no transporte, baixo custo, ausência de patógenos, riqueza de nutrientes e condições adequadas a crescimento e desenvolvimento da planta (Silva et al., 2001). A partir do processo de compostagem se obtém substrato de baixo custo que fornece à planta sustentação, macro e micronutrientes, aumento na capacidade de troca de cátions e uniformidade de estabelecimento de plântulas (Kiehl, 2012).

$\mathrm{O}$ reaproveitamento de resíduos é benéfico, uma vez que, evita os efeitos negativos, que os mesmos trazem quando são descartados de forma inadequada no meio ambiente. Quando são destinados de forma ambientalmente correta, a exemplo da compostagem, (Brasil, 2010) contribuem para o desenvolvimento sustentável, pois, representam economia de recursos, aproveitamento de resíduos, e consequentes ganhos ambientais (Dutra et al., 2013). Santos et al. (2014) afirma que hortaliças cultivadas com substrato produzido em compostagem escolar apresentam alto padrão de qualidade.

Acredita-se que além da utilização do produto final da compostagem escolar nos vegetais cultivados nas escolas, o material também possa ser utilizado no estabelecimento de mudas florestais, podendo, no futuro serem utilizadas em programas de educação ambiental e recomposição de áreas degradadas e de preservação ambiental. Essa pesquisa objetivou avaliar a emergência e crescimento inicial de plântulas de Copaifera pubiflora Benth. expostas à terra vegetal produzida em compostagem escolar.

\section{Material e Métodos}

O experimento foi desenvolvido entre os meses de janeiro e março de 2017, na sede da Empresa Brasileira de Pesquisa Agropecuária (Embrapa Roraima, Boa Vista). As sementes de copaíba foram coletadas do chão, abaixo da copa de sete matrizes em área de mata ciliar, no município de Boa Vista, Roraima, entre as coordenadas geográficas $2^{\circ} 52^{\prime} 15^{\prime \prime}$ e $2^{\circ} 52^{\prime} 17^{\prime}$ ' de latitude norte e $60^{\circ} 40^{\prime} 14^{\prime}$ ' e
60'40'23', de longitude oeste, sendo descartadas as sementes danificadas por insetos, doenças ou com má formação. Posteriormente, foram classificadas visualmente quanto ao tamanho (pequenas ou grandes) e realizada a caracterização biométrica (comprimento, diâmetro e massa da semente) utilizando um paquímetro digital $(0,01 \mathrm{~mm})$.

A terra vegetal utilizada para a realização do experimento foi oriunda de compostagem escolar, realizada por Marques et al. (2017) na Escola Municipal Menino de Jesus em Boa Vista, Roraima. As composteiras foram montadas para fins didáticos, no intuito de reduzir a destinação incorreta de resíduos orgânicos, tais como, restos de alimentos da cantina e podas do jardim, conscientizando e sensibilizando crianças do ensino básico.

A terra vegetal foi caracterizada, com a determinação dos seguintes parâmetros físico-químicos: umidade a $65^{\circ} \mathrm{C}$, capacidade de retenção de água, matéria orgânica total, condutividade elétrica, totais de sais dissolvidos e potencial hidrogeniônico. Utilizaram-se metodologias para resíduos orgânicos urbanos propostas por Kiehl (1985; 2012), com adaptações.

Após quarteamento manual, as amostras da terra vegetal foram colocadas em cápsulas de alumínio taradas, pesadas e levadas à estufa com ventilação forçada (Quimis), graduada para a faixa $60-65^{\circ} \mathrm{C}$, permanecendo por 24 horas, sendo pesadas após resfriamento.

Para determinação da capacidade de retenção de água utilizaram-se funil com papel de filtro qualitativo e proveta graduada $(100 \mathrm{~mL})$. O funil foi colocado sobre a proveta e adicionou-se $20 \mathrm{~g}$ do resíduo, em seguida, acrescentou-se lentamente, $50 \mathrm{~mL}$ de água deionizada. A capacidade de retenção de água foi determinada através da diferença entre o volume de água deionizado adicionado pela quantidade de água deionizada depositada na proveta, sendo os valores expressos em porcentagem.

A determinação da matéria orgânica total foi pelo método de perda por ignição (combustão). As amostras foram colocadas em cadinhos de porcelana calcinados, tarados e levados à estufa (Quimis) a $105-110^{\circ} \mathrm{C}$, por 4 horas. Em seguida, as amostras foram colocadas novamente em dessecador, posteriormente, foram levadas ao forno mufla (Quimis) por 2 horas. Após resfriamento completo da mufla, retiraram-se os cadinhos, colocando-os em dessecador e após total resfriamento dos cadinhos, pesaram-se as amostras.

As mensurações de condutividade elétrica, totais de sais dissolvidos e potencial hidrogeniônico foram realizadas em extrato homogêneo da diluição de 10 gramas de amostra em $100 \mathrm{~mL}$ de água deionizada $(1: 10 \mathrm{~m} / \mathrm{v})$. A homogeneização das amostras foi realizada manualmente, com auxílio de bastão de vidro, durante aproximadamente, 60 segundos por amostra.

A condutividade elétrica e totais sais dissolvidos foram mensurados a partir de condutivímetro microprocessado (Quimis, modelo Q-405M), calibrado com solução padrão de $\mathrm{KCl}$ com $1418 \mu \mathrm{S} \mathrm{cm} \mathrm{cm}^{-1}$. O potencial hidrogeniônico foi mensurado utilizando-se potenciômetro digital (Lucadema, modelo LUCA-210), calibrado com soluções tampões de potencial hidrogeniônico de 4,0 e 7,0. 
A areia foi adquirida no comércio local e uniformizada granulometricamente a $0,8 \mathrm{~mm}$ com auxílio de uma peneira. Posteriormente, foi lavada com água e esterilizada, a fim de eliminar microrganismos presentes. A esterilização foi realizada durante 2 horas, em estufa (Quimis) graduada na faixa $200 \pm 10{ }^{\circ} \mathrm{C}$, seguindo recomendações das Regras para Análises de Sementes (Brasil, 2009).

Para a acomodação de substratos, utilizaram-se três bandejas de polipropileno $(58,2 \times 41,0 \times 16,5 \mathrm{~cm})$ com 54 tubetes cada. Cada tubete com volume de $300 \mathrm{~cm}^{3}$, furo e quatro estrias verticais. Realizou-se a limpeza e higienização dos recipientes com detergente neutro, e submersão em solução a $2 \%$ de hipoclorito de sódio (NaClO) por 30 minutos. Logo em seguida, foram lavadas em água corrente e deixados para secar sob sol direto por meia hora.

Para formulação dos tratamentos, utilizaram-se diferentes doses de terra vegetal, com nove repetições cada, sendo um tubete a unidade experimental (semeando-se uma semente em cada). Os tratamentos e suas composições são descritos na Tabela 1.

Tabela 1. Identificação e descrição das doses terra vegetal (TV) e areia esterilizada (AE) utilizados no experimento

\begin{tabular}{cc}
\hline Tratamento & Composição \\
\hline D1 & $0 \% \mathrm{TV}+100 \% \mathrm{AE}$ \\
D2 & $20 \% \mathrm{TV}+80 \% \mathrm{AE}$ \\
D3 & $40 \% \mathrm{TV}+60 \% \mathrm{AE}$ \\
D4 & $60 \% \mathrm{TV}+40 \% \mathrm{AE}$ \\
D5 & $80 \% \mathrm{TV}+20 \% \mathrm{AE}$ \\
D6 & $100 \% \mathrm{TV}+0 \% \mathrm{AE}$ \\
\hline
\end{tabular}

O experimento foi realizado em ambiente protegido, em um galpão de alvenaria com telado metálico, que reduz a insolação e a entrada de animais. Foi semeada uma semente por tubete a $1 \mathrm{~cm}$ de profundidade. As variáveis avaliadas 40 dias após semeadura foram porcentagem de emergência, altura da parte aérea, diâmetro do coleto e matéria seca total.

A porcentagem de emergência foi avaliada segundo as Regras para Análises de Sementes (Brasil, 2009), tendo a primeira contagem aos 9 dias e a última aos 33 dias. A altura da parte aérea das plântulas foi mensurada com régua milimetrada, medido no nível do substrato até o ápice da plântula. $\mathrm{O}$ diâmetro do coleto foi mensurado utilizando paquímetro digital $(0,01 \mathrm{~mm})$, medindo a $0,5 \mathrm{~cm}$ da superfície do substrato. A matéria seca de total da planta foi determinada com auxílio de balança de precisão $(0,001 \mathrm{~g})$ e estufa de circulação de ar quente, graduada na faixa de $65 \pm 5$ ${ }^{\circ} \mathrm{C}$, realizando a pesagem após peso constante da amostra.

O delineamento experimental foi inteiramente casualizado, em arranjo fatorial $6 \times 2$, com nove repetições. Os tratamentos constaram da interação de seis doses de terra vegetal e com dois tamanhos de sementes de copaíba. Os dados foram analisados estatisticamente por meio de análise de variância (ANOVA), regressões lineares e polinomiais e teste de Tukey, a 5\% de probabilidade. Utilizou-se o programa computacional SISVAR ${ }^{\circledR}$ (Ferreira, 2011.

\section{Resultados e discussão}

A caracterização físico-química do resíduo orgânico produzido está apresentada na Tabela 2. De acordo com esses resultados, o resíduo estudado não atendeu aos critérios exigido pela instrução normativa brasileira (Brasil, 2009) e padrão teórico vigente (Kiehl, 2012) para ser classificado como resíduos bioestabilizado, devido as variáveis de capacidade de retenção de água e matéria orgânica total estarem fora do padrão exigido. Dessa maneira o resíduo foi considerado como terra vegetal. Contudo, o resíduo apresenta umidade a $65{ }^{\circ} \mathrm{C}$, condutividade elétrica, totais de sais dissolvidos e potencial hidrogeniônico desejáveis para fertilizantes orgânicos, estes três últimos, demonstrando que possivelmente seu uso não ocasionará problemas com salinidade, alcalinidade na maioria das culturas agrícolas (Ayers; Westcost, 1985).

Tabela 2. Caracterização físico-química de terra vegetal produzida a partir de compostagem de escola de Boa Vista, Roraima

\begin{tabular}{ccccccc}
\hline Substrato & U65 (\%) & CRA (\%) & MOT $(\%)$ & CE $\left(\mathrm{dS} \mathrm{m}^{-1}\right)$ & TSD $\left(\mathrm{mg} \mathrm{L}^{-1}\right)$ & $\mathrm{pH}$ \\
\hline TV & $23,0 \pm 0,57$ & $40,4 \pm 0,50$ & $33,2 \pm 0,58$ & $0,83 \pm 0,01$ & $591,6 \pm 9,86$ & $6,9 \pm 0,05$ \\
IN/PT & $<50,0$ & $>80,0$ & $>40,0$ & $<4,0$ & $<2.560,0$ & $>6,0$ \\
\hline
\end{tabular}

$\mathrm{TV}=$ terra vegetal; IN/PT $=$ instrução normativa/padrão teórico; U65 = umidade a $65^{\circ} \mathrm{C}$; CRA = capacidade de retenção de água; $\mathrm{MOT}=$ matéria orgânica total; $\mathrm{CE}=$ condutividade elétrica; $\mathrm{TSD}=$ totais de sais dissolvidos; $\mathrm{pH}=\mathrm{potencial}$ hidrogeniônico.

As sementes coletadas no Rio Branco (bairro Caçari) se assemelham a sementes de frutos de $C$. officinalis coletados em áreas de mata na margem direita do rio Branco, aproximadamente $12 \mathrm{~km}$ ao Sul da cidade de Boa Vista, Roraima, avaliadas por Andrade Jr. (1998) com média de comprimento, largura, espessura e massa de sementes de 13 $\mathrm{mm}, 9 \mathrm{~mm}, 8 \mathrm{~mm}, 0,8 \mathrm{~g}$, respectivamente.

A $C$. multijuga apresenta sementes um pouco maiores quando comparadas entre as espécies, com valores de 20 $\mathrm{mm}$ de comprimento, $13 \mathrm{~mm}$ de largura, $11 \mathrm{~mm}$ de espessura e peso de 1,8 g (Brum et al., 2007).

Os resultados médios da biométria e das variáveis experimentais de sementes de $C$. pubiflora estão descritos nas Tabelas 3 e 4, respectivamente.

Houve significância entre o tamanho de sementes e a altura de plantas, sendo a altura de sementes grandes as que 
apresentaram os maiores valores, variando de 11,5 (D6) a $13,1 \mathrm{~cm}$ (D1). Enquanto que as plantas oriundas de sementes pequenas, tiveram alturas médias entre 10,5 (D1) e $12 \mathrm{~cm}$ (D3).

Tabela 3. Comprimento ( $\mathrm{mm})$, diâmetro (mm) e massa (g) de sementes pequenas (P) e grandes (G) de Copaifera pubiflora Benth. coletadas em área natural de Boa Vista, Roraima.

\begin{tabular}{ccccccc}
\hline Medida & \multicolumn{2}{c}{ Comprimento } & \multicolumn{2}{c}{ Diâmetro } & \multicolumn{2}{c}{ Massa } \\
\cline { 2 - 7 } & $\mathrm{P}$ & $\mathrm{G}$ & $\mathrm{P}$ & $\mathrm{G}$ & $\mathrm{P}$ & $\mathrm{G}$ \\
\hline Média & 11,43 & 14,04 & 7,92 & 8,68 & 0,49 & 0,72 \\
DP & 0,51 & 0,66 & 0,55 & 0,48 & 0,05 & 0,08 \\
Máximo & 12,25 & 15,96 & 9,97 & 9,88 & 0,63 & 0,95 \\
Mínimo & 9,98 & 12,60 & 6,76 & 7,18 & 0,39 & 0,60 \\
CV & 4,49 & 4,68 & 6,97 & 5,58 & 10,80 & 10,89 \\
\hline
\end{tabular}

$\overline{\mathrm{CV} \% \text { - percentual do coeficiente de variação; DP - Desvio padrão. }}$
Não houve diferença significativa no diâmetro de coleto em função das doses de terra vegetal. Entretanto, observouse significância entre tamanhos de sementes, onde as plantas originárias de sementes grandes tiveram média máxima de 2,37 cm (D3), enquanto as de pequenas tiveram média máxima de $2,12 \mathrm{~cm}$ (D4).

Altura de planta e diâmetro do coleto aqui reportados assemelham-se com os resultados de Vieira e Weber (2015) que testaram doses de Basaplant ${ }^{\circledR}$ Cambissolo húmico de textura franco-arenosa no cultivo de $C$. langsdorffii, e registraram para plantas com 120 dias de idade entre 13,5 e $17,7 \mathrm{~cm}$ de altura, com diâmetro de coleto entre 2,0 a 2,9 mm. Já Augusto et al. (2003) reportaram valores para a altura de planta entre 11,6 e $14,5 \mathrm{~cm}$, e diâmetro de coleto entre 3,7 e 3,8 mm, utilizando fertirrigações convencional e água residuária nesta mesma espécie (C. langsdorffii).

Tabela 4. Valores médios de emergência de plântulas $(\%)$, altura de planta $(\mathrm{cm})$, diâmetro de coleto (mm) e matéria seca total (mg) de planta de $C$. pubiflora cultivadas em função de doses de terra vegetal e do tamanho de sementes 40 dias após semeadura

\begin{tabular}{|c|c|c|c|c|c|c|c|c|}
\hline \multirow{2}{*}{ Dose } & \multicolumn{2}{|c|}{ Emergência de plântula } & \multicolumn{2}{|c|}{ Altura de planta } & \multicolumn{2}{|c|}{ Diâmetro do coleto } & \multicolumn{2}{|c|}{ Matéria seca total de planta } \\
\hline & SP & SG & SP & SG & SP & SG & SP & SG \\
\hline 0 & $\begin{array}{c}100,0 \pm 0,00^{\mathrm{aA}} \\
(0,0 \%)\end{array}$ & $\begin{array}{c}100,0 \pm 0,00^{\mathrm{aA}} \\
(0,0 \%)\end{array}$ & $\begin{array}{c}10,5 \pm 0,50^{\mathrm{bB}} \\
(4,8 \%)\end{array}$ & $\begin{array}{c}13,1 \pm 0,42^{\mathrm{aA}} \\
(3,2 \%)\end{array}$ & $\begin{array}{c}1,78 \pm 0,03^{\mathrm{aB}} \\
(1,9 \%)\end{array}$ & $\begin{array}{c}2,25 \pm 0,16^{\mathrm{aA}} \\
(7,2 \%)\end{array}$ & $\begin{array}{c}309,7 \pm 1,25^{\mathrm{aB}} \\
(0,4 \%)\end{array}$ & $\begin{array}{c}421,5 \pm 4,43^{\mathrm{aA}} \\
(1,1 \%)\end{array}$ \\
\hline 20 & $\begin{array}{c}88,9 \pm 9,27^{\mathrm{bB}} \\
(10,4 \%)\end{array}$ & $\begin{array}{c}100,0 \pm 0,00^{\mathrm{aA}} \\
(0,0 \%)\end{array}$ & $\begin{array}{c}11,5 \pm 0,50^{\mathrm{aB}} \\
(4,3 \%)\end{array}$ & $\begin{array}{c}12,7 \pm 0,26^{\mathrm{aA}} \\
(2,1 \%)\end{array}$ & $\begin{array}{c}1,87 \pm 0,03^{\mathrm{aB}} \\
(1,9 \%)\end{array}$ & $\begin{array}{c}2,35 \pm 0,13^{\mathrm{aA}} \\
(5,5 \%)\end{array}$ & $\begin{array}{c}271,5 \pm 4,41^{\mathrm{aB}} \\
(1,6 \%)\end{array}$ & $\begin{array}{c}350,5 \pm 10,11^{\mathrm{aA}} \\
(2,9 \%)\end{array}$ \\
\hline 40 & $\begin{array}{c}88,9 \pm 7,81^{\mathrm{bA}} \\
(8,79 \%)\end{array}$ & $\begin{array}{c}88,9 \pm 6,00^{\mathrm{bA}} \\
(6,8 \%)\end{array}$ & $\begin{array}{c}12,0 \pm 0,43^{\mathrm{aA}} \\
(3,6 \%)\end{array}$ & $\begin{array}{c}12,5 \pm 0,71^{\mathrm{aA}} \\
(5,7 \%)\end{array}$ & $\begin{array}{c}2,03 \pm 0,08^{\mathrm{aB}} \\
(4,1 \%)\end{array}$ & $\begin{array}{c}2,37 \pm 0,12^{\mathrm{aA}} \\
(5,2 \%)\end{array}$ & $\begin{array}{c}259,7 \pm 5,75^{\mathrm{aB}} \\
(2,2 \%)\end{array}$ & $\begin{array}{c}305,7 \pm 1,25^{\mathrm{bA}} \\
(0,4 \%)\end{array}$ \\
\hline 80 & $\begin{array}{c}66,7 \pm 7,07^{\mathrm{cB}} \\
(10,6 \%)\end{array}$ & $\begin{array}{c}77,8 \pm 6,66^{\mathrm{cA}} \\
(8,6 \%)\end{array}$ & $\begin{array}{c}11,5 \pm 0,82^{\mathrm{aA}} \\
(7,1 \%)\end{array}$ & $\begin{array}{c}11,7 \pm 0,56^{\mathrm{bA}} \\
(4,8 \%)\end{array}$ & $\begin{array}{c}2,10 \pm 0,03^{\mathrm{aB}} \\
(1,4 \%)\end{array}$ & $\begin{array}{c}2,32 \pm 0,05^{\mathrm{aA}} \\
(2,0 \%)\end{array}$ & $\begin{array}{c}255,5 \pm 2,31^{\mathrm{aB}} \\
(0,9 \%)\end{array}$ & $\begin{array}{c}345,2 \pm 7,12^{\mathrm{aA}} \\
(2,1 \%)\end{array}$ \\
\hline 100 & $\begin{array}{c}44,4 \pm 5,27^{\mathrm{dB}} \\
(11,8 \%)\end{array}$ & $\begin{array}{c}77,8 \pm 4,40^{\mathrm{cA}} \\
(5,7 \%)\end{array}$ & $\begin{array}{c}11,3 \pm 0,75^{\mathrm{aA}} \\
(6,7 \%)\end{array}$ & $\begin{array}{c}11,5 \pm 0,32^{\mathrm{bA}} \\
(2,8 \%)\end{array}$ & $\begin{array}{c}1,95 \pm 0,04^{\mathrm{aB}} \\
(2,0 \%)\end{array}$ & $\begin{array}{c}2,19 \pm 0,02^{\mathrm{aA}} \\
(1,0 \%)\end{array}$ & $\begin{array}{c}302,7 \pm 2,58^{\mathrm{aB}} \\
(0,9 \%)\end{array}$ & $\begin{array}{c}376,5 \pm 0,79^{\mathrm{aA}} \\
0,2 \%)\end{array}$ \\
\hline $\begin{array}{l}\text { Equação } \\
\text { da } \\
\text { regressão } \\
\mathrm{R}^{2}\end{array}$ & $\begin{array}{c}\mathrm{y}=-0,5238 \mathrm{x}+ \\
102,11^{* * * *} \\
\mathrm{R}^{2}=0,9242\end{array}$ & $\begin{array}{c}\mathrm{y}=-0,254 \mathrm{x}+ \\
101,59^{* * *} \\
\mathrm{R}^{2}=0,9143\end{array}$ & $\begin{array}{c}\mathrm{y}=-0,0004 \mathrm{x}^{2} \\
+0,0484 \mathrm{x}+ \\
10,6^{* * * *} \\
\mathrm{R}^{2}=0,9160\end{array}$ & $\begin{array}{c}\mathrm{y}=9 \mathrm{E}-06 \mathrm{x}^{2}- \\
0,0172 \mathrm{x}+ \\
13,093^{* * * *} \\
\mathrm{R}^{2}=0,9913\end{array}$ & $\begin{array}{c}\mathrm{y}=-8 \mathrm{E}-05 \mathrm{x}^{2} \\
+0,0105 \mathrm{x}+ \\
1,7471^{* * *} \\
\mathrm{R}^{2}=0,9219\end{array}$ & $\begin{array}{c}\mathrm{y}=-6 \mathrm{E}-05 \mathrm{x}^{2} \\
+0,0054 \mathrm{x}+ \\
2,2539^{* * *} \\
\mathrm{R}^{2}=0,9737\end{array}$ & $\begin{array}{c}\mathrm{y}=0,0208 \mathrm{x}^{2}- \\
2,2044 \mathrm{x}+ \\
309,98^{* * *} \\
\mathrm{R}^{2}=0,9287\end{array}$ & $\begin{array}{c}\mathrm{y}=0,0327 \mathrm{x}^{2}- \\
3,5715 \mathrm{x}+ \\
414,42^{* * * *} \\
\mathrm{R}^{2}=0,8968\end{array}$ \\
\hline
\end{tabular}

SP - Sementes pequenas; SG - Sementes grandes. Médias seguidas de mesma letra minúscula na coluna e, maiúscula na linha, não diferem estatisticamente entre si, pelo teste ANOVA, seguido do teste de Tukey a 5\% de probabilidade. CV = coeficiente de variação. *** parâmetros altamente significativos.

Não houve diferença significativa das doses de terra vegetal acrescida em areia esterilizada na matéria seca total de C. pubiflora. Observou-se diferença apenas no tamanho de sementes, quando as sementes grandes proporcionaram plantas com média máxima de 421,5 mg (D1) e as sementes pequenas, originaram plantas com média máxima de $309,7 \mathrm{mg}$ (D1). Plântulas obtidas de sementes grandes apresentaram matéria seca total maior que as obtidas de sementes pequenas.

Porém, todos os tratamentos aqui avaliados apresentaram valores de matéria seca total inferiores quando comparados com os da literatura, cujos resultados variaram de 830 e 990 $\mathrm{mg}$ (Augusto et al., 2003), 860 e $1.860 \mathrm{mg}$ (Vieira e Weber, 2015) e entre 1.704 e $1.907 \mathrm{mg}$ (Dutra et al., 2015) em plantas de $C$. langsdorffii, sendo que os três estudos aqui citados foram mensurados após os 120 dias de semeadura, o que pode justificar esta superioridade.

\section{Conclusões}

Houve diferença significativa na qualidade de mudas de $C$. pubiflora cultivadas em função do tamanho de sementes e doses de terra vegetal. As plantas oriundas de sementes grandes apresentam melhor qualidade nas variáveis mensuradas.

$\mathrm{O}$ uso de terra vegetal em doses maiores que 20 e $40 \%$ interferem negativamente na emergência de sementes pequenas e grandes de $C$. pubiflora respectivamente. O crescimento inicial de plantas de $C$. pubiflora é beneficiado quando se utiliza doses de até $60 \%$ de terra vegetal e $40 \%$ de areia esterilizada, para ambos tamanhos de sementes.

A terra vegetal produzida no processo de compostagem escolar nas condições de Boa Vista, Roraima, além de ser utilizada para cultivo de hortas e jardins, pode ser incorporada a composição de substratos para espécies florestais, estimulando a 
emergência e crescimento inicial de plântulas, desde que, seja conhecida a dose ideal para cada espécie.

\section{Referências}

Andrade Jr., M.A. 1998. Sementes de Copaifera officinalis L. Caesalpiniaceae: uma abordagem autoecológica, fisiológica e tecnológica. Dissertação de mestrado. Instituto Nacional de Pesquisas da Amazônia: Manaus: 114p.

Augusto, D.C.C.; Guerrini, I.A.; Engel, V.L.; Rouseau, G.X. 2003. Utilização de esgotos domésticos tratados através de um sistema biológico na produção de mudas de Croton floribundus Spreng. (capixingui) e Copaifera langsdorffii Desf. (copaíba). Árvore, 27(3): 335-342.

Ayers, R.S.; Westcost, D.W. 1985. Water quality for agriculture. FAO, Roma, $174 \mathrm{p}$.

Brasil. 2009. Instrução Normativa SDA N ${ }^{\circ} 25$, de 23 de julho de 2009. Brasília: Ministério da Agricultura Pecuária e Abastecimento.

Brasil. 2010. Lei n ${ }^{\circ}$ 12.305, de 2 de agosto de 2010. Brasília, DF: [s.n]. Disponível em: http://www.planalto.gov.br/ccivil_03/_ato20072010/2010/lei/112305.htm. Acesso em: abril de 2017.

Brasil. 2009. Ministério da Agricultura, Pecuária e Abastecimento. Regras para análise de sementes. Brasília: Mapa/ACS, 399 p.

Brum, H.D.; Mesquita, M.R.; Ferraz, I.D. K. 2007. Descrição Comparativa dos Propágulos e Plântulas de Copaifera multijuga Hayne e Copaifera officinalis Jacq. (Fabaceae). Biociências, Porto Alegre, 5(1):351-353.

Dutra, T.R.; Massad, M.D.; Sarmento, F.Q.; Oliveira, J.C. 2013. Substratos alternativos e métodos de quebra de dormência para a produção de mudas de canafístula. Ceres, 60(1): 72-78.

Dutra, T.R.; Grazziotti, P.H.; Santana, R.C.; Massad, M.D. 2015. Qualidade de mudas de copaíba produzidas em diferentes substratos e níveis de sombreamento. Floresta, 45(3): 635-644.

Ferreira, D. F. 2011. Sisvar: a computer statistical analysis system. Ciência e Agrotecnologia, 35(6): 1039-1042.

Jeromini, T.S.; Fachinelli, R.; Silva, G.Z.; Pereira, S.T.S. 2017. Emergência de plântulas e crescimento inicial de copaíba sob diferentes substratos. Pesquisa Florestal Brasileira, 37(90): 219-223.

Kiehl, E. J. 1985. Fertilizantes Orgânicos. $1^{a}$ ed. Editora Agronômica Ceres Ltda., Piracicaba, 492p.

Kiehl, E.J. 2012. Manual de compostagem: maturação e qualidade do composto. $6^{\mathrm{a}}$ ed. Agronômica Ceres Ltda., Piracicaba, 171p.

Marques, C.S.; Pereira, M.J.C.; Guimarães, P.V.P.; Araújo, S.L.F. 2017. Experiência de educação ambiental em escola pública de Boa Vista-RR: conhecendo e aproveitando resíduos escolares. In: Aguiar, W. J. De; Eldeir, S. G; Bezerra, R. P. L. (2. ed.). Resíduos sólidos: abordagens práticas em educação ambiental. v.1. Editora Universitária da Universidade Federal Rural de Pernambuco, Recife, Pernambuco, p. 6473.

Martins-da-Silva, R.C., Pereira, J.F., Lima, H.C. 2008. O gênero Copaifera (Leguminosae - Caesalpinioideae) na Amazônia brasileira. Rodriguésia, 59(3): 455-476.

Pereira, R.S.; Ranal, M.; Dorneles, M.C.; Santana, D.G.; Borges, K.C.F.; Carvalho, M.P. 2007. Emergência de plântulas de Copaifera langsdorffii Desf. Revista Brasileira de Biociências, 5(2): 1005-1007.

Ros, C.O.; Rex, F.E.; Ribeiro, I.R.; Kafer, P.S.; Rodrigues, A.C.; Silva, R.F.; Somavilla, L. 2015. Uso de substrato compostado na produção de mudas de Eucalyptus dunnii e Cordia trichotoma. Floresta e Ambiente, 22(4): 549-558.

Santos, C.B.; Longhi, S.J.; Hoppe, J.M.; Moscovich, F.A. 2000. Efeito do volume de tubetes e tipos de substratos na qualidade mudas de Cryptomeria japonica (L.F.) D. Don. Ciência Florestal, 10(2): 1-15.

Santos, A.M.L.; Martins, R.M.L.; Souza, R.D.; Mota, R.M.F.; Fernandes, C.T. 2014. Incentivo ao uso da compostagem de resíduos sólidos em uma horta escolar do município de Jaciara-MT. Unopar Científica: Ciências Humanas e Educação, 15(0): 321-329.

Silva, R.P.; Peixoto, J.R.; Junqueira, N.T.V. 2001. Influência de diversos substratos no desenvolvimento de mudas de maracujazeiro azedo (Passiflora edulis Sims f. flavicarpa DEG). Fruticultura, 23(2): 377-381.

Shanley, P.; Leite, A.; Alechandre, A.; Azevedo, C. 2005. Copaíba. In Shanley, P., Medina, G. (EDS.). Frutíferas e plantas úteis na vida amazônica. $1^{\mathrm{a}}$ ed. CIFOR/ Imazon, Belém, 300p.

Varela, V.P.; Costa, S.S.; Ramos, M. B.P. 2005. Influência da temperatura e do substrato na germinação de sementes de itaubarana (Acosmium nitens (Vog.) Yakovlev) -Leguminosae, Caesalpinoideae. Acta Amazonica, Manaus, 35(1): 35-39.

Veiga Junior, V.F.; Pinto, A.C. 2002. O gênero Copaifera L. Química Nova, 25(2): 273-286, 2002

Vieira, R.F.; Silva, S.R. 2002. Estratégias para conservação e manejo de recursos genéticos de plantas medicinais e aromáticas: resultados da $1^{\mathrm{a}}$ reunião técnica. Embrapa Recursos Genéticos e Biotecnologia, Instituto Brasileiro do Meio Ambiente e dos Recursos Naturais Renováveis (IBAMA), Conselho Nacional de Desenvolvimento Científico e Tecnológico (CNPq), Brasília, p.146-150.

Vieira, C.R.; Weber, O.L. dos S. 2015. Influência do substrato na produção de mudas de espécies medicinais. Nativa, 3(2): 135-142. 